\title{
$\pi$-extended porphyrin dimers as efficient near-infrared emitters and two-photon absorbers
}

Julie Schmitt, ${ }^{[a]}$ Valérie Heitz, ${ }^{[a]^{*}}$ Sébastien Jenni, ${ }^{[a]}$ Angélique Sour, ${ }^{[a]}$ Frédéric Bolze, ${ }^{[\mathrm{b}]^{*}}$ Barbara Ventura ${ }^{[\mathrm{c}]^{*}}$

${ }^{[a]}$ Laboratoire de Synthèse des Assemblages Moléculaires Multifonctionnels, Institut de Chimie de Strasbourg, CNRS/UMR 7177, Université de Strasbourg, 4, rue Blaise Pascal, 67000 Strasbourg (France).E-mail:v.heitz@unistra.fr

[b] CAMB, UMR 7199, UdS/CNRS, Faculté de Pharmacie, Université de Strasbourg, 74 route du Rhin,67401 Illkirch (France).E-mail: frederic.bolze@unistra.fr

[c] Istituto ISOF-CNR, Via P. Gobetti 101, 40129 Bologna (Italy). E-mail: barbara.ventura@isof.cnr.it 


\title{
$\pi$-extended porphyrin dimers as efficient near-infrared emitters and two-photon absorbers
}

\begin{abstract}
A Zn-porphyrin dimer and its linear conjugate with two diketopyrrolopyrrole (DPP) units have been prepared and their one- and two-photon photophysical properties have been characterized. The already wide conjugation of the dimer is further extended to the DPP containing derivative, leading to absorption and emission features in the near infra-red. The fluorescence quantum yield of the two compounds is of the order of 0.20 in organic solvents, a remarkable value for NIR emitting Zn-porphyrin systems. High two-photon absorption cross sections of $10000 \mathrm{GM}$ and $21500 \mathrm{GM}$ are measured at $910 \mathrm{~nm}$ for the dimer and the DPP conjugate, respectively, making these dimers promising for two-photon excited fluorescence imaging applications.
\end{abstract}

Keywords: porphyrin; diketopyrrolopyrrole; near-infrared emitter; two-photon absorption

Subject classification codes: photochemistry, organic chemistry

\section{Introduction}

Porphyrins are appealing compounds for a wide range of applications, in particular those related to bio-medical chemistry thanks to their close affinity to naturally occurring pigments and catalytic components.

Extension of the conjugation of the porphyrin aromatic system has been shown to be an interesting tool to decrease the HOMO-LUMO gap and develop near-infrared (NIR) absorbing and emitting materials (1-5). The development of efficient NIR emitters finds important applications in bio-medical imaging $(6,7)$, due to the high transparency of biological environments to NIR radiation. One of the main issues in the development of NIR probes is the drop of the emission quantum yield with the decrease of the energy of 
the emitting state, due to the increased efficiency of non-radiative deactivation processes (energy gap law) (8).

We recently showed that conjugation of diketopyrrolopyrrole (DPP) units to a $\mathrm{Zn}$ porphyrin component leads to NIR absorption and emission in the larger derivatives, with the interesting and unusual trend of an increase in the fluorescence quantum yield with the decrease of the emission energy (3). DPP-porphyrin conjugates, moreover, have been found to be efficient two-photon absorbers (3, 9-11), with exceptional potential in imaging and sensing applications, two-photon photodynamic therapy and theranostics.

We further explore the topic here, by describing the preparation and the characterization of a $\mathrm{Zn}$-porphyrin dimer and of a linear derivative containing two DPP units linked by ethynyl spacers. Detailed photophysical investigations of the compounds have been performed, together with the analysis of their two-photon absorption activity. Fluorescence spectra and quantum yields, combined with two-photon absorption crosssection, are discussed in view of assessing the potential of the two novel compounds as biphotonic NIR fluorophores.

\section{Results and discussion}

The chemical structures of the compounds of the present study, ZnP-ZnP and DPP-ZnPZnP-DPP, are represented in Figure 1. They consist of a butadiyne-linked Zn(II) porphyrin dimer linked to TIPS-protected ethynyl groups for $\mathbf{Z n P - Z n P}$ or conjugated to two peripheral diketopyrrolopyrrole (DPP) units through ethynyl spacers for DPP-ZnPZnP-DPP. Several hydrophilic di- and tri-ethylene glycol chains on the central porphyrin core and on peripheral DPPs enhance the water solubilization of the compounds. 

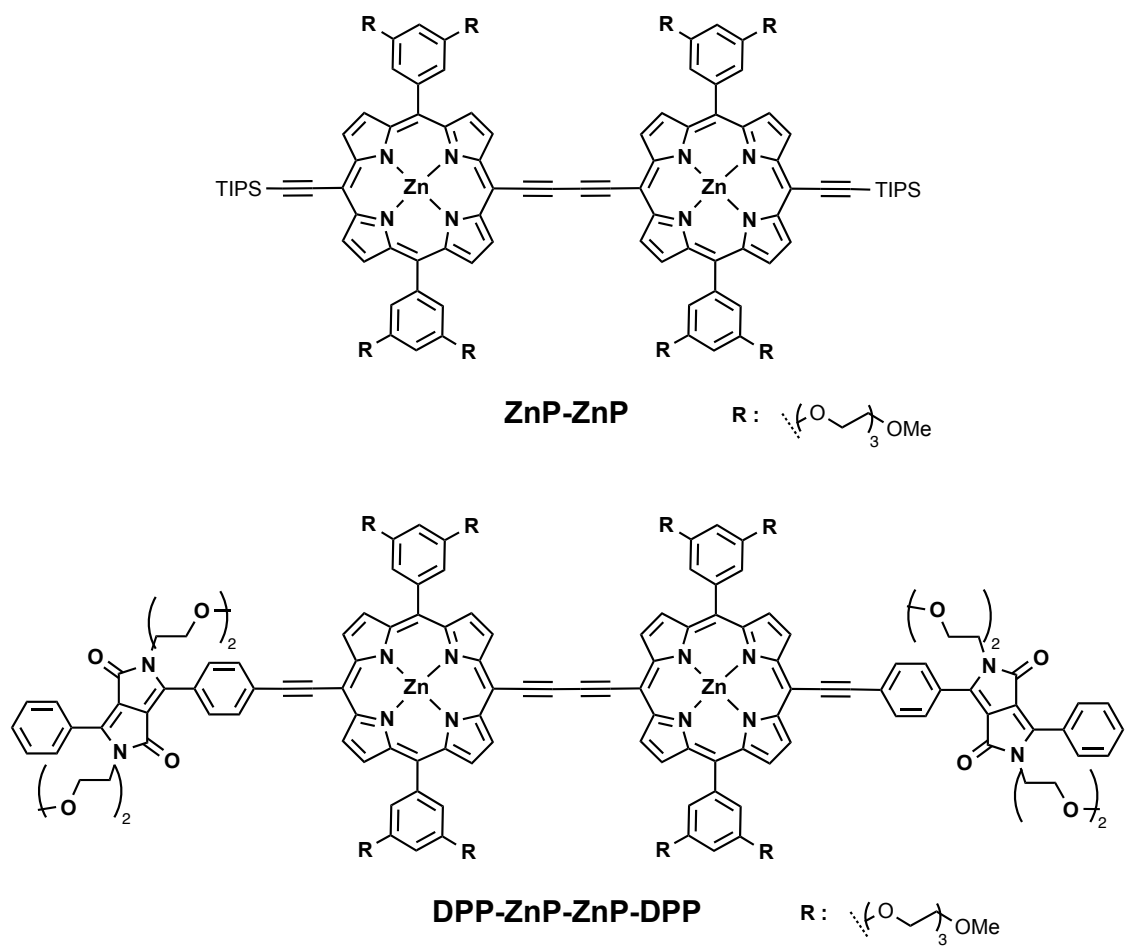

Figure 1. Chemical structure of ZnP-ZnP and DPP-ZnP-ZnP-DPP.

\section{Synthesis}

ZnP-ZnP and DPP-ZnP-ZnP-DPP were obtained by $\mathrm{Pd} / \mathrm{Cu}$-catalyzed homocoupling reaction (12) of ZnP-TIPS (9) and DPP-ZnP $(9,11)$ respectively (Figure 2). The reaction proceeded at room temperature and was completed after $45 \mathrm{~min}$. Both compounds have been purified by silica gel column chromatography and obtained with $81 \%$ and $80 \%$ yield, respectively. They were fully characterized by NMR spectroscopy (see Figures S1-S2 for ZnP-ZnP and Figures S4-S5 for DPP-ZnP-ZnP-DPP). ESI mass spectrometry of ZnP$\mathbf{Z n P}$ and DPP-ZnP-ZnP-DPP showed the isotopic profile of the molecular ionic species $[\mathrm{M}+\mathrm{Na}]^{+}$and $[\mathrm{M}+2 \mathrm{Na}]^{2+} / 2$ respectively that were in accordance with the calculated profile (Figures S3 and S6). 

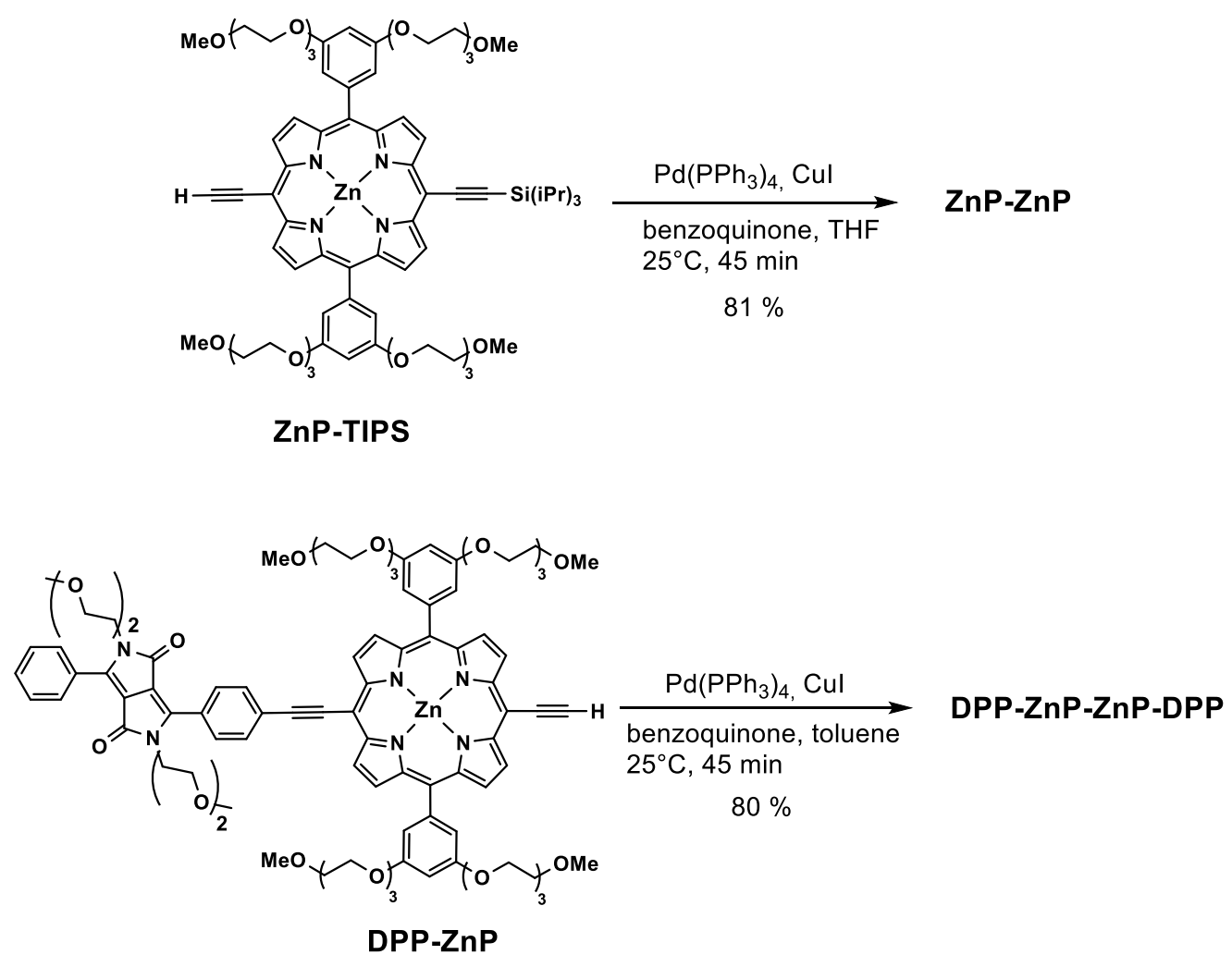

Figure 2. Synthesis of ZnP-ZnP and DPP-ZnP-ZnP-DPP.

\section{Absorption and emission properties. Singlet oxygen production}

The photophysical characterization of ZnP-ZnP and DPP-ZnP-ZnP-DPP was performed in two organic solvents, dichloromethane (DCM) and dimethyl sulfoxide (DMSO), and an aqueous solution $\left(\mathrm{H}_{2} \mathrm{O}\right.$ added with $1 \%$ of DMSO).

Absorption spectra of the two dimers in DCM are reported in Figure 3 and those recorded in DMSO and $\mathrm{H}_{2} \mathrm{O}+1 \%$ DMSO are reported in Figures $\mathrm{S} 7$ and $\mathrm{S} 8$, respectively. A splitting of the Soret band is observed, indicative of strong electronic interactions between the two porphyrins (13-15). The Q-band region extends in the near-infrared, in particular for the DPP containing porphyrin dimer which absorbs up to $800 \mathrm{~nm}$. The bathochromic shift observed for DPP-ZnP-ZnP-DPP with respect to ZnP-ZnP, of the order of 3-4 $\mathrm{nm}$ in the Soret region and of ca. $20 \mathrm{~nm}$ in the Q-bands in DCM and DMSO (in $\mathrm{H}_{2} \mathrm{O}+1 \%$ DMSO these values increase to ca. $10 \mathrm{~nm}$ and $50 \mathrm{~nm}$, respectively) is 
ascribed to the enhanced conjugation introduced by the ethynyl linked DPP units $(3,16$, 17). For each compound, a shift of the absorption bands to lower energies is observed when the polarity of the solvent increases $\left(\mathrm{DCM}<\mathrm{DMSO}<\mathrm{H}_{2} \mathrm{O}\right)$ and the extent of the shift is higher for the DPP containing dimer (Table 1), indicating a contribution from charge-transfer transitions (3).

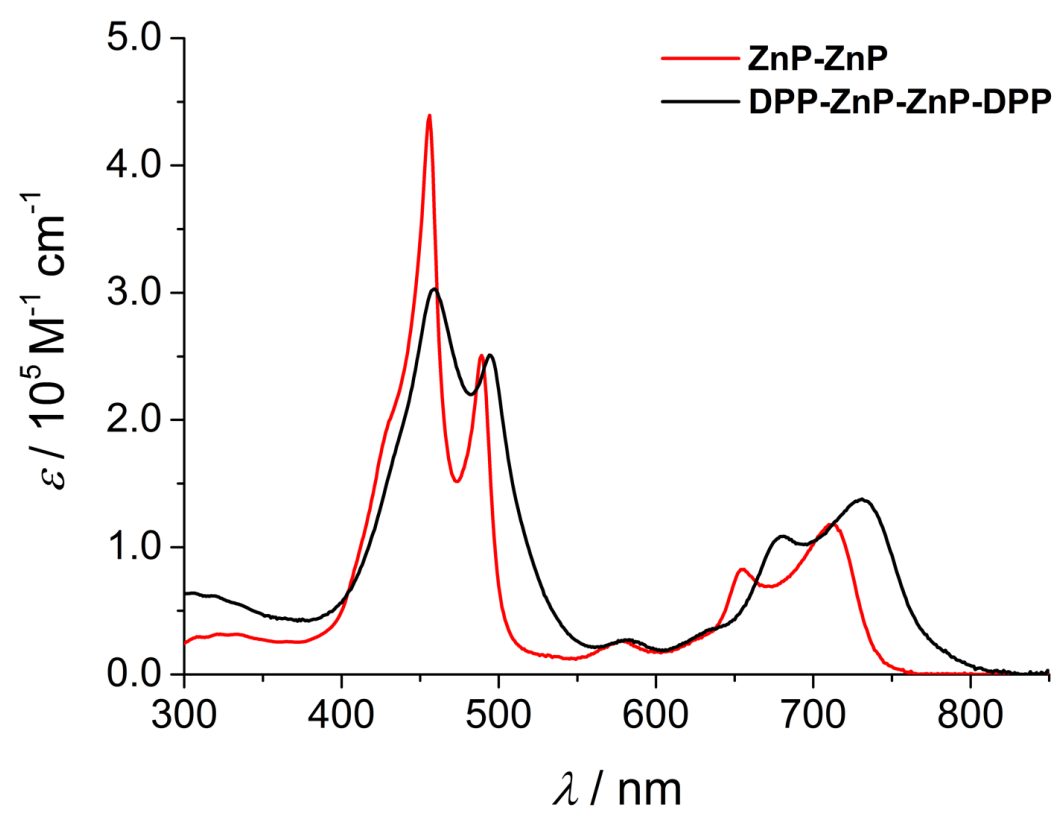

Figure 3. Absorption spectra of ZnP-ZnP and DPP-ZnP-ZnP-DPP in DCM.

The room temperature emission spectra of the two compounds in DCM are reported in Figure 4; those recorded in DMSO and $\mathrm{H}_{2} \mathrm{O}+1 \%$ DMSO are shown in Figures S9 and S10. The relevant emission parameters are collected in Table 1. The fluorescence of the two dimers is in the NIR region, with a pronounced shift to lower energies when the polarity of the solvent increases (Table 1). The fluorescence quantum yield is of the order of 0.20 in the two organic solvents, higher than that of related DPP- Zn-porphyrin conjugates containing only one porphyrin component (3). The significant value of the fluorescence quantum yield is interesting in consideration of the low energy of the 
transitions. This result, in apparent contradiction with the "energy gap law", can be ascribed to the increased value of the oscillator strength brought by the conjugation ( 3 , 18) between the two porphyrin units in $\mathbf{Z n P - Z n P}$ and the extended conjugation towards the DPP units in DPP-ZnP-ZnP-DPP. This is reflected in the remarkable value of the radiative rate constant, of the order of $2 \times 10^{8} \mathrm{~s}^{-1}$ for both compounds (Table 1).

Measurements in DCM added with $1 \%$ of pyridine revealed a red-shift of the absorption spectra by $3-5 \mathrm{~nm}$ in the Soret region and $15-20 \mathrm{~nm}$ in the Q region (see Figures S11a and S12a) and a parallel red-shift of the emission spectra by ca. $20 \mathrm{~nm}$ (Figures S11b and S12b) with fluorescence quantum yields almost unaltered with respect to pure DCM (Table 1), the latter excluding the occurrence of aggregation phenomena in the pure solvent.

On the other hand, in the aqueous medium, solubility and aggregation issues are proven by the lower, but still significant, emission quantum yields of the compounds (of the order of $10^{-3}$ ) and by short or multi-exponential decays (Table 1).

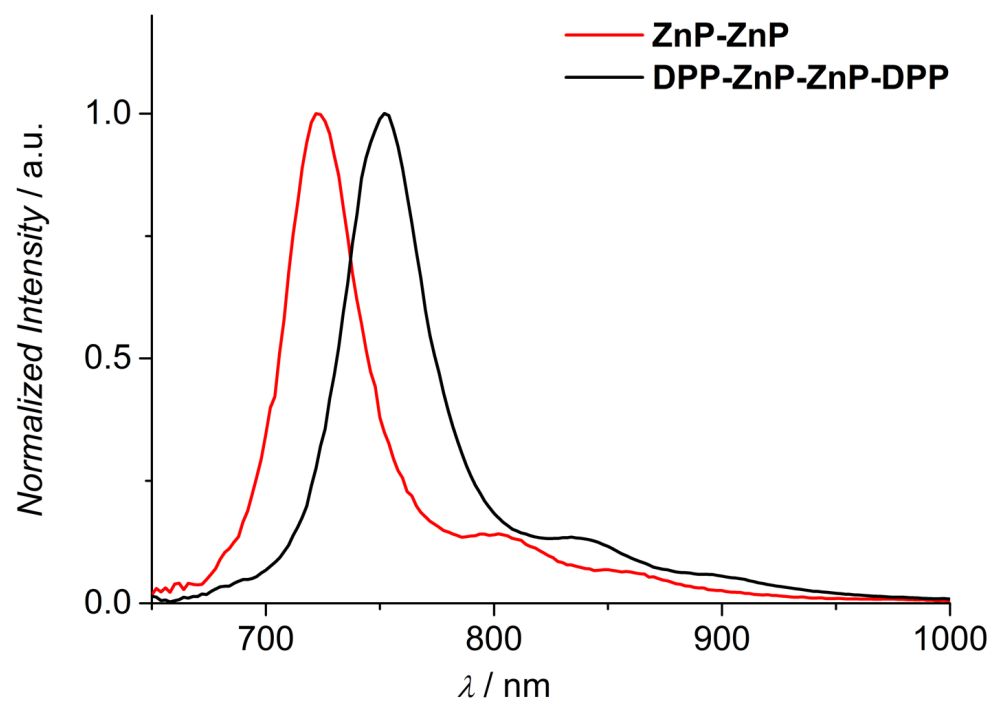

Figure 4. Normalized corrected emission spectra of ZnP-ZnP and DPP-ZnP-ZnP-DPP in DCM.

Table 1. Photophysical data and singlet oxygen production quantum yields at room temperature in the different solvents. 
In brackets, values obtained with $1 \%$ pyridine. ${ }^{a}$ From corrected emission spectra. ${ }^{b}$ Fluorescence quantum yields, measured with reference to DPP-ZnP-DPP in aerated

\begin{tabular}{|c|c|c|c|c|c|c|c|}
\hline & & $\lambda_{\max } / \mathrm{nm}^{a}$ & $\phi_{\mathrm{fl}}{ }^{b}$ & $\tau / \mathrm{ns}^{c}$ & $K_{\mathrm{r}} / \mathrm{s}^{-1}$ & $K_{\mathrm{nr}} / \mathrm{s}^{-1}$ & $\phi_{\Delta}{ }^{d}$ \\
\hline ZnP-ZnP & DMSO & $742,826,880 \mathrm{sh}$ & 0.21 & 1.22 & $1.7 \times 10^{8}$ & $6.5 \times 10^{8}$ & 0.38 \\
\hline \multirow[t]{2}{*}{ DPP-ZnP-ZnP-DPP } & DCM & $752,838,898$ sh $(774,862,922 \mathrm{sh})$ & $0.23(0.24)$ & $1.21(1.25)$ & $1.9 \times 10^{8}$ & $6.4 \times 10^{8}$ & 0.11 \\
\hline & DMSO & $774,862,916$ sh & 0.20 & 0.95 & $2.1 \times 10^{8}$ & $8.4 \times 10^{8}$ & 0.19 \\
\hline
\end{tabular}

$\operatorname{DCM}\left(\phi_{\mathrm{fl}}=0.16\right)(9) .{ }^{\mathrm{c}}$ Fluorescence lifetimes, excitation at 465 and $560 \mathrm{~nm} .{ }^{\mathrm{d}}$ Singlet oxygen production quantum yields, see the Experimental Section for details. ${ }^{\mathrm{e}}$ With $1 \%$ DMSO. ${ }^{\mathrm{f}}$ A longer component (5.2 ns) is detected for $20 \%$ of the decay.

Low temperature investigations have been performed at $77 \mathrm{~K}$ in a DCM:MeOH (1:1) glassy mixture. Fluorescence spectra, shown in Figure S13, are slightly red-shifted with respect to the room temperature case (ca. $10 \mathrm{~nm}$, Table S1 and Table 1). The energies of the lowest singlet states, derived from the emission maxima, are $1.69 \mathrm{eV}$ and $1.63 \mathrm{eV}$ for ZnP-ZnP and DPP-ZnP-ZnP-DPP, respectively. Phosphorescence could not be detected at low temperature, even upon addition of an external heavy atom, i.e. $50 \%$ of ethyl iodide in the same mixture.

In order to better characterize the triplet states of the two dimers, laser flashphotolysis experiments in air-free DMSO have been performed, as well as singlet oxygen determination. Transient absorption spectra are shown in Figure 5 and absorption maxima and lifetimes are reported in Table S2. The spectra show ground state bleaching features in the 400-800 $\mathrm{nm}$ region and broad absorption bands that extend far in the NIR. A bathochromic shift of the spectra is observed when moving from $\mathbf{Z n P - Z n P}$ to the DPP containing dimer, a trend already observed with the introduction of DPP units in DPPporphyrin conjugates and ascribed to the increased delocalization brought by the ethynyl linked DPP elements. 


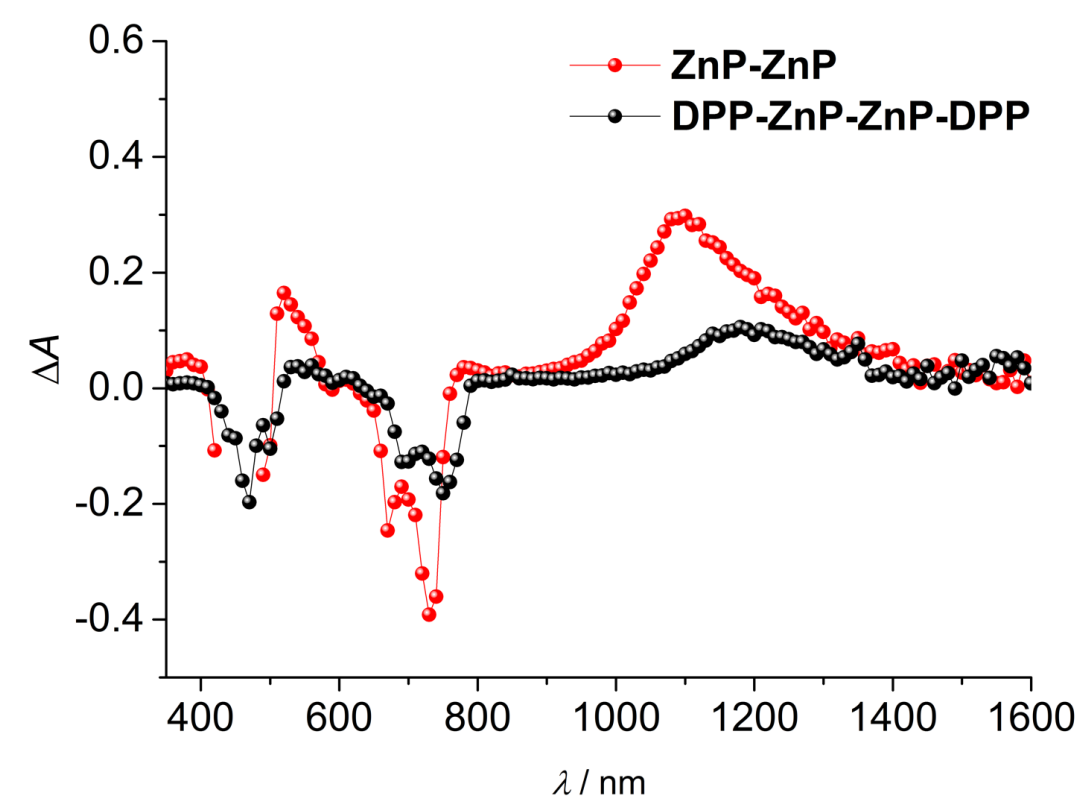

Figure 5. End of pulse transient absorption spectra of air-free DMSO solutions of ZnP-ZnP and DPP-ZnP-ZnP-DPP upon excitation at $532 \mathrm{~nm}\left(\mathrm{E}=3 \mathrm{~mJ}\right.$ per pulse, $A_{532}$ $=0.2)$.

Singlet oxygen quantum yield determinations for the two dimers have been performed by direct measurement of singlet oxygen phosphorescence in DCM (9) and by means of an indirect method in DMSO. The latter was made necessary by the poor luminescence properties of singlet oxygen in DMSO (19). Direct measurements in $\mathrm{H}_{2} \mathrm{O}$ (or $\mathrm{D}_{2} \mathrm{O}$ ) $+1 \%$ DMSO were precluded by the limited solubility of the compounds in the aqueous solvent. Singlet oxygen phosphorescence spectra from optically matched solutions of the two dimers and the standard Rose Bengal bis(triethyl-ammonium) salt $\left(\phi_{\Delta}=0.48\right)(20)$ in DCM are reported in Figure S14. The measured values of $\phi_{\Delta}$ are 0.37 and 0.11 for ZnP-ZnP and DPP-ZnP-ZnP-DPP, respectively (Table 1). Indirect determinations in DMSO made use of DPBF as a singlet oxygen trap and ZnPc as a standard $\left(\phi_{\Delta}=0.67\right)(21)$. Changes in the absorption spectra of mixtures of DPBF and 
the examined compounds (or the standard) upon irradiation at $646 \mathrm{~nm}$ are reported in Figure S15. By comparison of the DPBF degradation rates (see the Experimental Section for details), $\phi_{\Delta}$ values of 0.38 and 0.19 for $\mathbf{Z n P - Z n P}$ and DPP-ZnP-ZnP-DPP, respectively, were derived (Table 1). These values are very close to those obtained in DCM and confirm a decrease of efficiency in singlet oxygen production with the introduction of the DPP units in the dimeric structure. This result can be ascribed to a decrease in energy of the triplet excited state of the DPP containing dimer, which should follow the same trend observed for the lowest singlet excited state, due to the enhanced conjugation of the system. By considering the singlet-triplet energy gaps observed for a series of DPP-porphyrin conjugates (3), we can estimate the energy of the triplet excited state of DPP-ZnP-ZnP-DPP to be of the order of $1.0 \mathrm{eV}$, thus very close to the singlet oxygen level $(0.98 \mathrm{eV})$. This explains the low efficiency of the T-T energy-transfer process and, accordingly, the reduced singlet oxygen sensitization observed for the extended dimer.

\section{Two-photon absorption}

Two-photon absorption (TPA) properties of ZnP-ZnP and DPP-ZnP-ZnP-DPP were determined by the way of their two-photon induced fluorescence in DCM/1\% pyridine. Both compounds show strong TPA with a cross section $\left(\sigma_{2}\right)$ maximum at $910 \mathrm{~nm}$ of 10000 and 21500 GM for ZnP-ZnP and DPP-ZnP-ZnP-DPP respectively (Figure 6). The TPA properties of the silyl protected dimer are close to the one reported in DCM/1\% pyridine for a similar porphyrin dimer bearing a 3,5-diterbutyl-phenyl group in meso position and with hexyl groups on the protecting silyl moiety (9100 GM) (22). The increase in the conjugation length and the introduction of the electro-active DPP system on each side of the porphyrin dimer induce a strong increase of the $\sigma_{2}$, up to $21500 \mathrm{GM}$ at $910 \mathrm{~nm}$. This effect is similar to the one obtained by ionic electron accepting moieties 
such as a methyl pyridinium. The TPA maximum is also red-shifted by $30 \mathrm{~nm}$ compared to a carboxylic acid salt bearing system with a similar $\pi$ backbone (16).

The use of a porphyrin dimer instead a single porphyrin macrocycle is highly valuable as the $\sigma_{2}$ of a bis-DPP porphyrin system showed maximum TPA cross-section of $4000 \mathrm{GM}$ in the $900 \mathrm{~nm}$ region $(3,9)$.

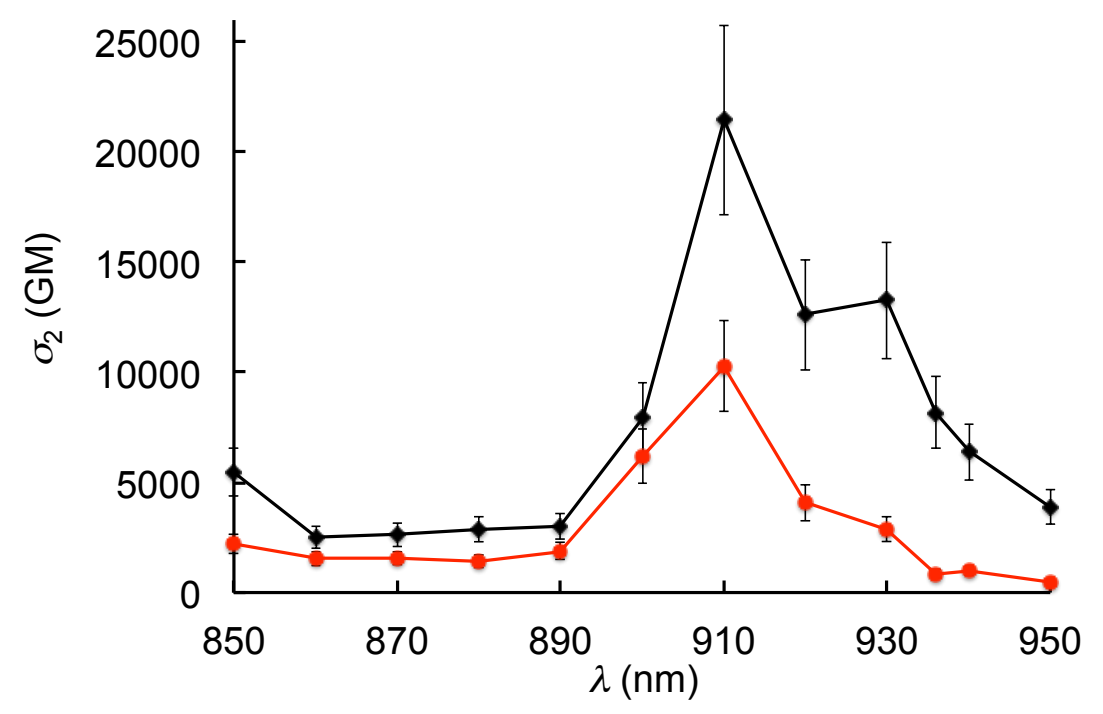

Figure 6. Two-photon excitation spectra at $10^{-5} \mathrm{M}$ of $\mathbf{Z n P - Z n P}$ (red) and DPP-ZnP-ZnPDPP (black) in DCM/1\% pyridine.

\section{Conclusions}

The synthesis of two $\pi$-extended porphyrin dimers ZnP-ZnP and DPP-ZnP-ZnP-DPP was successfully realized based on a $\mathrm{Pd} / \mathrm{Cu}$ homocoupling reaction of porphyrin functionalized with a terminal alkyne.

The two compounds show absorption and fluorescence features in the nearinfrared, the latter extending up to $1000 \mathrm{~nm}$ for the longer array. Interestingly, they present a fluorescence quantum yield of 0.20 in organic solvents, a remarkable value for NIR emitting Zn-porphyrin compounds. 
Triplet excited state absorption spectra show a bathochromic shift with the introduction of the DPP units, extending in the NIR up to $1500 \mathrm{~nm}$. A decrease in the singlet oxygen quantum yield from ca. 0.40 to ca. 0.15 is observed and ascribed to the decrease of the triplet energy level due to the extended conjugation of the larger system. As a general observation, a low efficiency in singlet oxygen generation, which could prevent application of the material in photodynamic therapy, has to be taken into account when designing systems with fluorescence far in the NIR region.

A remarkable value of TPA cross-section of $10000 \mathrm{GM}$ at $910 \mathrm{~nm}$ in DCM $+1 \%$ pyridine is found for the porphyrin dimer, which even doubles to $21500 \mathrm{GM}$ for the DPPdimer conjugate. This leads to two-photon brightness $\left(\sigma_{2} \phi_{\mathrm{fl}}\right)$ values of the order of 2000 and $4300 \mathrm{GM}$ at $910 \mathrm{~nm}$ in organic solvents for ZnP-ZnP and DPP-ZnP-ZnP-DPP, respectively. The two explored compounds, and the DPP conjugated dimer in particular, can thus be considered promising candidates for imaging applications. These results open the way to the design of novel probes based on conjugated porphyrin systems.

\section{Experimental section}

\section{Synthesis}

Details on the synthesis of the compounds are presented in the ESI Section.

\section{One-photon spectroscopy and photophysics}

DCM and DMSO were of spectroscopic grade from Merck and Carlo Erba, respectively. Tri-distilled (Millipore Milli-Q) $\mathrm{H}_{2} \mathrm{O}$ was used. 1,3-diphenylisobenzofuran (DPBF), Znphthalocyanine (ZnPc), pyridine and Rose Bengal bis(triethyl-ammonium) salt were from Aldrich. Ethyl iodide was from Alfa Aesar. 
Absorption spectra were recorded with a Perkin-Elmer Lambda 650 UV-Vis spectrophotometer. Emission spectra were collected with a FLS920 fluorimeter (Edinburgh) equipped with a Hamamatsu R5509-72 InP/InGaAs photomultiplier tube supercooled at $193 \mathrm{~K}$ in a liquid nitrogen cooled housing and a TM300 emission monochromator with a NIR grating blazed at $1000 \mathrm{~nm}$ (sensitivity range: $300-1700 \mathrm{~nm}$ ). The spectra have been corrected for the wavelength dependent phototube response. The fluorescence quantum yields have been determined with reference to DPP-ZnP-DPP in aerated DCM $\left(\phi_{\mathrm{fl}}=0.16\right)(9)$. Fluorescence lifetimes have been measured with an IBH Time Correlated Single Photon Counting apparatus with excitation both at $465 \mathrm{~nm}$ and $560 \mathrm{~nm}$. The analysis of the luminescence decay profiles against time was accomplished with the DAS6 Decay Analysis Software provided by the manufacturer. Experiments at $77 \mathrm{~K}$ in frozen glasses made use of quartz capillary tubes immersed in liquid nitrogen contained in a home-made quartz dewar. Estimated errors are $10 \%$ on exponential lifetimes, $20 \%$ on quantum yields, $20 \%$ on molar absorption coefficients and $3 \mathrm{~nm}$ on emission and absorption peaks.

Nanosecond laser flash photolysis experiments made use of systems based on Nd:YAG lasers: a JK laser (18 ns pulse duration, $532 \mathrm{~nm}, 3 \mathrm{~mJ} /$ pulse, UV-Vis detection) and a Surelite Continuum laser (6 ns pulse duration, $532 \mathrm{~nm}, 3 \mathrm{~mJ} / \mathrm{pulse}$, UV-Vis-NIR detection), using a right-angle analysis on the excited sample (23). The samples were bubbled with argon for ca. $15 \mathrm{~min}$ and sealed in homemade $10 \mathrm{~mm}$ optical path length cells.

Singlet oxygen production quantum yields in DCM have been measured in air equilibrated solutions with reference to Rose Bengal bis(triethyl-ammonium)salt $\phi_{\Delta}=$ 0.48 in DCM) (20), by comparing the areas of singlet oxygen phosphorescence spectra measured with the NIR fluorimeter described above. Since the singlet oxygen emission 
spectrum overlapped with the tail of fluorescence of the two examined compounds (see Figure S12) the latter has been subtracted from the spectra prior to derive the area of the singlet oxygen signal. Excitation at $442 \mathrm{~nm}$ has been performed with a Kimmon Koha Co., Ltd. HeCd laser (with power reduced to ca. $7 \mathrm{~mW}$ ). Singlet oxygen production quantum yields in DMSO have been measured by means of a comparative method using DPBF as a singlet oxygen trap, which is oxidized by singlet oxygen and its degradation can be monitored by the decrease of its absorbance (24). Zinc phthalocyanine (ZnPc) has been used as a standard $\left(\phi_{\Delta}=0.67\right)(21)$. Solutions of the standard or of the compound containing DPBF $2.2 \times 10^{-5} \mathrm{M}$, prepared in the dark, have been irradiated at $646 \mathrm{~nm}\left(A_{646}\right.$ $\sim 0.3$ ) by using an irradiation set-up composed by a $150 \mathrm{~W}$ Xenon lamp (LOT) and a Omni- $\lambda 150$ monochromator (Zolix), under continuous stirring. The light intensity was 6 $\mathrm{mW} / \mathrm{cm}^{2}$. The singlet oxygen quantum yield of the sample $\left(\phi_{\Delta}\right)$ has been determined by comparing the DPBF degradation rates for the sample and the standard, as described previously (3). The absorption spectrum of DPBF at each irradiation step has been derived by subtracting the constant absorption contribution of the examined compound from the spectrum of the mixture. The decrease of DPBF absorption has been followed at $400 \mathrm{~nm}$, a wavelength chosen to avoid distortion effects due to the high absorbance of some solutions around $450 \mathrm{~nm}$.

\section{Two-photon spectroscopy}

The two-photon excitation spectra were obtained by upconverted fluorescence method using a Ti:sapphire femtosecond laser Insight DS with pulse width $<120$ fs and a repetition rate of $80 \mathrm{MHz}$ (Spectra-Physics) as described previously (25). Briefly, the excitation beam was collimated over the cell length $(10 \mathrm{~mm})$ and the fluorescence, collected at $90^{\circ}$ of the excitation beam, was focused into an optical fiber connected to a 
spectrometer. The incident beam intensity was adjusted to ensure an intensity-squared dependence of the fluorescence over the whole spectral range investigated. Deviation from a two-photon process was detected below $830 \mathrm{~nm}$. Calibration of the spectra was performed by comparison with the published rhodamine B TPA spectrum (26).

\section{Acknowledgements}

The icFRC (http://www.icfrc.fr), LabEx CSC, and Region Alsace are gratefully acknowledged for a fellowship to J.S., and the Ministry of Education and Research is acknowledged for a Ph.D. fellowship to S.J.. Italian CNR (Project "PHEEL"), MIURCNR project "Nanomax" N-CHEM, CNR-ASRT (Egypt) Bilateral Cooperation Project "FLUO-NanoFAB" and H2020-MSCA-IF-2016-751175 project "SmartMOFs" are gratefully acknowledged.

\section{References}

(1) Duncan, T. V.; Susumu, K.; Sinks, L. E.; Therien, M. J. J. Am. Chem. Soc. 2006, 128, 9000-9001.

(2) Hoffmann, M.; Karnbratt, J.; Chang, M. H.; Herz, L. M.; Albinsson, B.; Anderson, H. L. Angew. Chem.-Int. Edit. 2008, 47, 4993-4996.

(3) Alam, M. M.; Bolze, F.; Daniel, C.; Flamigni, L.; Gourlaouen, C.; Heitz, V.; Jenni, S.; Schmitt, J.; Sour, A.; Ventura, B. Phys. Chem. Chem. Phys. 2016, 18, 2195421965.

(4) Bonifazi, D.; Scholl, M.; Song, F. Y.; Echegoyen, L.; Accorsi, G.; Armaroli, N.; Diederich, F. Angew. Chem.-Int. Edit. 2003, 42, 4966-4970.

(5) Cho, H. S.; Jeong, D. H.; Cho, S.; Kim, D.; Matsuzaki, Y.; Tanaka, K.; Tsuda, A.; Osuka, A. J. Am. Chem. Soc. 2002, 124, 14642-14654.

(6) Yuan, L.; Lin, W. Y.; Zheng, K. B.; He, L. W.; Huang, W. M. Chem. Soc. Rev. 2013, 42, 622-661.

(7) Barbieri, A.; Bandini, E.; Monti, F.; Praveen, V. K.; Armaroli, N. Top. Curr. Chem. 2016, 374.

(8) Englman, R.; Jortner, J. Mol. Phys. 1970, 18, 145-164.

(9) Schmitt, J.; Heitz, V.; Sour, A.; Bolze, F.; Ftouni, H.; Nicoud, J. F.; Flamigni, L.; Ventura, B. Angew. Chem.-Int. Edit. 2015, 54, 169-173.

(10) Nowak-Krol, A.; Grzybowski, M.; Romiszewski, J.; Drobizhev, M.; Wicks, G.; Chotkowski, M.; Rebane, A.; Gorecka, E.; Gryko, D. T. Chem. Commun. 2013, 49, 83688370 . 
(11) Schmitt, J.; Heitz, V.; Sour, A.; Bolze, F.; Kessler, P.; Flamigni, L.; Ventura, B.; Bonnet, C. S.; Toth, E. Chem.-Eur. J. 2016, 22, 2775-2786.

(12) Williams, V. E.; Swager, T. M. J. Polym. Sci. Pol. Chem. 2000, 38, 4669-4676.

(13) Piet, J. J.; Taylor, P. N.; Wegewijs, B. R.; Anderson, H. L.; Osuka, A.; Warman, J. M. J. Phys. Chem. B 2001, 105, 97-104.

(14) Susumu, K.; Duncan, T. V.; Therien, M. J. J. Am. Chem. Soc. 2005, 127, 51865195 .

(15) Huang, T. H.; Chen, Y. J.; Lo, S. S.; Yen, W. N.; Mai, C. L.; Kuo, M. C.; Yeh, C. Y. Dalton Trans. 2006, 2207-2213.

(16) Kuimova, M. K.; Collins, H. A.; Balaz, M.; Dahlstedt, E.; Levitt, J. A.; Sergent, N.; Suhling, K.; Drobizhev, M.; Makarov, N. S.; Rebane, A.; Anderson, H. L.; Phillips, D. Org. Biomol. Chem. 2009, 7, 889-896.

(17) Tanaka, T.; Osuka, A. Chem. Soc. Rev. 2015, 44, 943-969.

(18) Ziessel, R.; Rihn, S.; Harriman, A. Chem.-Eur. J. 2010, 16, 11942-11953.

(19) Wilkinson, F.; Brummer, J. G. J. Phys. Chem. Ref. Data 1981, 10, 809-1000.

(20) Lamberts, J. J. M.; Schumacher, D. R.; Neckers, D. C. J. Am. Chem. Soc. 1984, 106, 5879-5883.

(21) Ogunsipe, A.; Chen, J.-Y.; Nyokong, T. New J. Chem. 2004, 28, 822-827.

(22) Drobizhev, M.; Stepanenko, Y.; Dzenis, Y.; Karotki, A.; Rebane, A.; Taylor, P. N.; Anderson, H. L. J. Phys. Chem. B 2005, 109, 7223-7236.

(23) Flamigni, L. J. Phys. Chem. 1992, 96, 3331-3337.

(24) Seotsanyana-Mokhosi, I.; Kuznetsova, N.; Nyokong, T. J. Photochem. Photobiol. A 2001, 140, 215-222.

(25) Hayek, A.; Ercelen, S.; Zhang, X.; Bolze, F.; Nicoud, J. F.; Schaub, E.; Baldeck, P. L.; Mely, Y. Bioconjugate Chem. 2007, 18, 844-851.

(26) Makarov, N. S.; Drobizhev, M.; Rebane, A. Opt. Express 2008, 16, 4029-4047. 